dards of safety and efficacy. Provided that such a back door remains closed, a more user-friendly system of expanded access as described by Lurie et al. may help reduce pressure for more problematic measures currently being considered by Congress that would substantially lower the drug-approval standards of the agency. ${ }^{3}$ If the new FDA procedures enable patients with lifethreatening conditions who lack adequate therapeutic options and who do not qualify for ongoing clinical trials to access potentially beneficial experimental treatments through expandedaccess protocols, it would remove a main ethical justification for the excessive acceleration of formal FDA approval of drugs before they can be adequately tested.
Ameet Sarpatwari, J.D., Ph.D. Jonathan J. Darrow, S.J.D., J.D. Aaron S. Kesselheim, M.D., J.D. Brigham and Women's Hospital Boston, MA

Since publication of their article, the authors report no further potential conflict of interest.

1. Guidance for industry: individual patient expanded access applications: Form FDA 3926. Silver Spring, MD: Food and Drug Administration (http://www.fda.gov/downloads/Drugs/Guidance ComplianceRegulatoryInformation/Guidances/UCM432717.pdf).

2. Quicker access to experimental drugs. New York Times. February 12, 2015:A28.

3. U.S. House of Representatives Energy and Commerce Committee. 21st Century Cures Act: discussion draft (http:/l energycommerce.house.gov/sites/republicans.energycommerce .house.gov/files/114/Analysis/Cures/20150127-Cures-Discussion -Document.pdf).

DOI: 10.1056/NEJMc1501823

\title{
PML in a Patient without Severe Lymphocytopenia Receiving Dimethyl Fumarate
}

TO THE EDITOR: Fumaric acid esters, which are prescribed for the treatment of psoriasis and multiple sclerosis, are considered to have a favorable risk profile. However, treatment-related progressive multifocal leukoencephalopathy (PML) has been described in association with long-lasting, severe lymphocytopenia ( $<500$ lymphocytes per cubic millimeter). ${ }^{1-3}$ This has led to the recommendation that lymphocyte counts should be monitored in patients receiving these drugs in order to prevent opportunistic infections such as PML. ${ }^{4}$ Here, we report a case of fatal PML after treatment with compounded dimethyl fumarate (DMF) in a patient without severe lymphocytopenia.

On July 18, 2014, a 64-year-old woman presented with a 2-week history of progressive apraxia. She had been receiving topical glucocorticoids and compounded delayed-release DMF (Psorinovo; compounding pharmacy, Mierlo-Hout) for the treatment of psoriasis since June 2012. Magnetic resonance imaging (MRI) of the brain showed multiple subcortical white-matter lesions (Fig. S1 in the Supplementary Appendix, available with the full text of this letter at NEJM.org). Leukocyte and lymphocyte counts were normal before DMF treatment but reached a nadir of 4000 cells and 792 cells per cubic millimeter, respectively, in June 2014 (Fig. 1). Analysis of the cerebrospinal fluid showed normal levels of leukocytes, protein, and glucose. The patient was seronegative for the human immunodeficiency virus. At that time, a diagnosis of PML was considered. However, testing of the cerebrospinal fluid for JC virus DNA on polymerase-chainreaction (PCR) assay was negative. Treatment with DMF was discontinued, and the patient received the diagnosis of atypical ischemic stroke.

Owing to progressive hemiparesis and somnolence, she was transferred to our hospital on August 14, 2014. Follow-up MRI showed a rapid and widespread dissemination of lesions suggestive of PML-immune reconstitution inflammatory syndrome (IRIS) (Fig. S1 in the Supplementary Appendix). Treatment with mefloquine, mirtazapine, and glucocorticoids was initiated. The patient's condition continued to deteriorate, and she died on August 26, 2014. PML was confirmed on histologic analysis of brain tissue (Fig. S2 in the Supplementary Appendix) and positive results on PCR assay for JC virus DNA in brain tissue and cerebrospinal fluid (see the Methods section in the Supplementary Appendix).

In our opinion, this case represents DMFassociated PML, since other immunosuppressive 


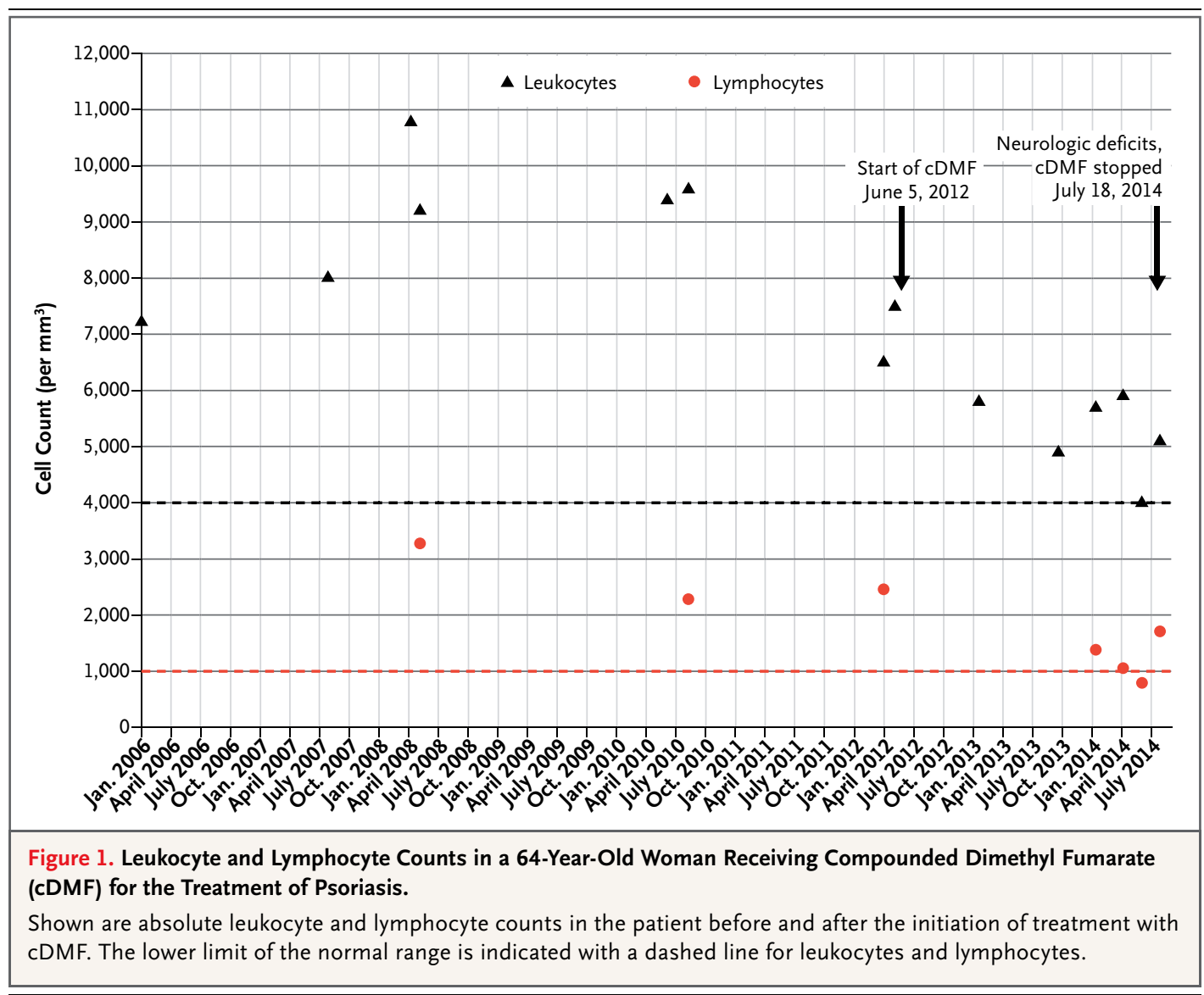

medications or coexisting medical conditions were absent. To our knowledge, this is the first reported case of compounded DMF-associated PML in a patient without severe lymphocytopenia, a situation that was previously thought to be unlikely. ${ }^{1,2,4}$ Since the number of patients who are being treated with DMF is rapidly increasing after approval of delayed-release DMF (Tecfidera) as first-line treatment for relapsing-remitting multiple sclerosis, our case raises important questions with respect to safety monitoring. Although more than 100,000 patients with multiple sclerosis have been treated with Tecfidera since 2013, the safety profile for long-term treatment is unknown. On October 22, 2014, the first case of PML in a patient receiving Tecfidera was reported; this patient had persistent, severe lymphocytopenia. ${ }^{3,5}$ Our case shows that PML can develop during treatment with compounded DMF in patients in whom reduced lymphocyte counts are less severe than those in cases that have been reported previously.

Dennis J. Nieuwkamp, M.D., Ph.D.

Jean-Luc Murk, M.D., Ph.D.

University Medical Center Utrecht

Utrecht, the Netherlands

d.nieuwkamp@umcutrecht.nl

Bob W. van Oosten, M.D., Ph.D.

VU University Medical Center

Amsterdam, the Netherlands

and Others

for the PML in Dutch MS Patients Consortium

A complete list of authors is available with the full text of this letter at NEJM.org.

Disclosure forms provided by the authors are available with the full text of this letter at NEJM.org.

1. Ermis U, Weis J, Schulz JB. PML in a patient treated with fumaric acid. N Engl J Med 2013;368:1657-8.

2. van Oosten BW, Killestein J, Barkhof F, Polman CH, Wattjes 
MP. PML in a patient treated with dimethyl fumarate from a compounding pharmacy. N Engl J Med 2013;368:1658-9. [Erratum, N Engl J Med 2013;368:1950.]

3. Food and Drug Administration. FDA warns about case of rare brain infection PML with MS drug Tecfidera (dimethyl fumarate). November 2014 (http://www.fda.gov/Drugs/DrugSafety/ ucm424625.htm).
4. Mrowietz U, Reich K. Case reports of PML in patients treated for psoriasis. N Engl J Med 2013;369:1080-1.

5. Rosenkranz T, Novas M, Terborg C. PML in a patient with lymphocytopenia treated with dimethyl fumarate. $\mathrm{N}$ Engl J Med 2015;372:1476-8.

DOI: 10.1056/NEJMc1413724

\section{PML in a Patient with Lymphocytopenia Treated with Dimethyl Fumarate}

TO THE EDITOR: We report the case of a 54-yearold woman with multiple sclerosis who was treated with delayed-release dimethyl fumarate (DMF; Tecfidera, Biogen Idec) and who died on October 13, 2014, from complications related to aspiration pneumonia and progressive multifocal leukoencephalopathy (PML) with severe, prolonged lymphocytopenia.

The patient, who had received the diagnosis of multiple sclerosis in 1996 and had been treated with glatiramer acetate, was randomly assigned to the placebo group in the 2-year Determination of the Efficacy and Safety of Oral Fumarate in Relapsing-Remitting Multiple Sclerosis (DEFINE) trial. ${ }^{1}$ She subsequently received delayed-release DMF (at a dose of $240 \mathrm{mg}$ three times daily) for 4.5 years in the open-label extension study. Twelve months after the initiation of delayedrelease DMF, severe lymphocytopenia (lymphocyte count, 290 to 580 cells per cubic millimeter) developed and persisted for 3.5 years.

Although the patient had no clinical disease activity since the first month of active treatment, she presented with new neurologic signs and symptoms consistent with a relapse in multiple sclerosis (severe gait disorder, speech disorder, and difficulties in left arm coordination) on August 11, 2014. Her condition did not improve with the administration of intravenous methylprednisolone (1 $\mathrm{g}$ once daily on August 11 to 13 , $2 \mathrm{~g}$ once daily on August 20 to 22, and 2 g once daily on September 20 to 22) and plasmapheresis for the suspected relapse ( 5 courses, 1 per day; September 26 to 30). Delayed-release DMF treatment was discontinued on August 23. PML was diagnosed on the basis of results on magnetic resonance imaging (Fig. S1 in the Supplementary Appendix, available with the full text of this letter at NEJM.org) and positive results on polymerase-chain-reaction assay for JC virus DNA in cerebrospinal fluid obtained by means of lumbar puncture on October 7. The patient had received no previous immunosuppressant drugs or natalizumab.

Since delayed-release DMF was approved in the United States in March 2013, more than 135,000 patients with multiple sclerosis have been treated, representing approximately 112,000

Figure 1 (facing page). Lymphocyte Counts in Patients with Multiple Sclerosis Receiving Delayed-Release Dimethyl Fumarate (DMF).

Shown are lymphocyte counts in studies involving patients with multiple sclerosis during treatment with delayed-release DMF. In the open-label extension of phase 3 trials, as of May 10 2014, the incidence of lymphocytopenia (lymphocyte count, $<500$ per cubic millimeter) was $1.9 \%$ (47 of 2470 patients) for 6 months or longer, $0.6 \%$ (15 of 2470 patients) for 2 years or longer, and $0.3 \%$ ( 8 of 2470 patients) for 3 years or longer. Panel A shows mean lymphocyte counts for 47 patients in whom consecutive post-baseline lymphocyte counts were less than 500 cells per cubic millimeter for at least 6 months, as compared with 2466 patients without lymphocytopenia. All the patients received delayed-release DMF at a dose of $240 \mathrm{mg}$ either two or three times daily. Counts were checked every 3 months in clinical trials, but values are shown for 6-month intervals for readability. The dashed line indicates the lower limit of the normal range (LLN). The I bars indicate standard errors. Panel B shows absolute lymphocyte counts over time in the same 47 DMF-treated patients, according to the daily dose and whether the patient was still receiving the drug. In the two dose groups ( $240 \mathrm{mg}$ administered either two or three times daily), similar proportions had a reduction in the lymphocyte count to below 800 cells per cubic millimeter or to below 500 cells per cubic millimeter. Post-dosing lymphocyte counts (available for 9 patients) generally increased in the 4 weeks after the discontinuation of treatment. 